\title{
In vitro reconstitution of interactions in the CARD9 signalosome
}

\author{
JIN HEE PARK* ${ }^{*}$ JAE YOUNG CHOI*, MIR FAISAL MUSTAFA* and HYUN HO PARK \\ School of Chemistry and Biochemistry and Graduate School of Biochemistry, \\ Yeungnam University, Gyeongsangnam 38541, Republic of Korea
}

Received August 9, 2016; Accepted February 27, 2017

DOI: $10.3892 / \mathrm{mmr} .2017 .7116$

\begin{abstract}
The caspase-associated recruitment domain (CARD)-containing protein 9 (CARD9) signalosome is composed of CARD9, B-cell CLL/lymphoma 10 (BCL10) and mucosa-associated lymphoid tissue lymphoma translocation protein 1 (MALT1). The CARD9 signalosome has been reported to exert critical functions in the immunoreceptor tyrosine-based activation motif-coupled receptor-mediated activation of myeloid cells, through nuclear factor- $\kappa \mathrm{B}$ pathways during innate immunity processes. During CARD9 signalosome assembly, BCL10 has been revealed to function as an adaptor protein and to interact with CARD9 via CARD-CARD interactions; BCL10 also interacts with MALT1 via its C-terminal Ser/Thr-rich region and the first immunoglobulin domain of MALT1. The CARD9 signalosome is implicated in critical biological processes; however, its structural and biochemical characteristics have yet to be elucidated. In the present study, CARD9 and BCL10 CARDs were successfully purified and characterized, and their biochemical properties were investigated. In addition, CARD9-BCL10 complexes were reconstituted in vitro under low salt and $\mathrm{pH}$ conditions. Furthermore, based on structural modeling data, a scheme was proposed to describe the interactions between CARD9 and BCL10. This provides a further understanding of the mechanism of how the CARD9 signalosome may be assembled.
\end{abstract}

\section{Introduction}

The transcription factor nuclear factor (NF)- $\kappa \mathrm{B}$ and mitogen-activated protein kinase (MAPK), which can be activated by several immunoreceptors, are crucial for immune cell activation, proliferation and survival (1-4). Genetic disorders

Correspondence to: Dr Hyun Ho Park, School of Chemistry and Biochemistry and Graduate School of Biochemistry, Yeungnam University, 280 Daehak-Ro, Gyeongsangnam 38541, Republic of Korea

E-mail: hyunho@ynu.ac.kr

*Contributed equally

Key words: innate immunity, NF-кB, CARD9 signalosome, death domain superfamily, CARD associated with mutations in NF- $\mathrm{B}$, or components of its signaling pathways, have been reported to cause numerous immune disorders, including autoimmunity and neoplastic disease $(5,6)$. The caspase-associated recruitment domain (CARD)-containing protein 9 (CARD9) signalosome, which is composed of CARD9, B-cell CLL/lymphoma 10 (BCL10) and mucosa-associated lymphoid tissue lymphoma translocation protein 1 (MALT1), is a major signaling complex that is important in immune cell processes, including myeloid cell-mediated NF- $\kappa \mathrm{B}$ activation (7-9).

CARD9 is a CARD-containing protein. The structure of its N-terminus is similar to that of caspase recruitment domain family member 11 , which is a member of the membrane-associated guanylate kinase family of scaffold proteins which has been reported to assist in the recruitment and assembly of signaling molecules in the cytoplasmic membrane $(8,10)$. CARD9 contains an N-terminal CARD domain followed by a coiled-coil domain (9). CARD domains belong to the class of protein motifs known as the death fold, which also contains death domains (DD), death effector domains (DED) and pyrin domains (PYD) (11-13). The death fold class of protein motifs is commonly identified in proteins implicated in apoptotic, inflammatory and immune signaling pathways (14-17). MALT1 contains an N-terminal $\mathrm{DD}$, followed by two immunoglobulin (Ig)-like domains and a C-terminal caspase-like domain. It has previously been reported that MALT1 ${ }^{-/-}$mice exhibited only mild defects in B-cell activation, thus suggesting that MALT1 may be dispensable. In addition, MALT1 has been reported to function as an E3 ubiquitin ligase, inducing K63-linked polyubiquitination of $\mathrm{NF}-\kappa \mathrm{B}$ essential modulator, which is a component of the inhibitor of $\kappa \mathrm{B}$ kinase complex that also functions as a protease capable of cleaving the deubiquitinating enzymes A20 and BCL10 (18-21). The proteolytic activity of MALT1 has been reported to be required for proper $\mathrm{NF}-\kappa \mathrm{B}$ activation, followed by interleukin (IL) -2 production $(20,21)$.

BCL10 is a member of the CARD-containing protein family and contains an N-terminal CARD and a C-terminal Ser/Thr-rich region. BCL10 has been reported to be able to induce apoptosis and activate NF- $\mathrm{B}$ (6). It has previously been demonstrated that during CARD9 signalosome assembly, BCL10 functioned as an adaptor protein, interacting with CARD9 via CARD-CARD interactions, and with MALT1 via its C-terminal Ser/Thr-rich region and the first Ig domain of MALT1 $(6,9,22,23)$. Upon myeloid cell activation, immunoreceptor tyrosine-based activation motif-coupled 
receptors, including Dectin-1, triggering receptor expressed on myeloid cells and Fc receptor, recognize antigens and activate downstream signaling events leading to the formation of stable CARD9 signalosome complexes that activate NF- $\mathrm{B}$ and MAPKs $(7,9)$. However, the exact molecular mechanisms implicated in CARD9 signalosome-mediated NF- $\mathrm{B}$ and MAPK activation have yet to be elucidated.

The CARD9 signalosome serves critical roles in immune receptor signaling events that govern innate and adaptive immunity; however, the insolubility of CARD-containing proteins under physiological conditions limits structural and biochemical studies (24-26). In the present study, the CARDs of CARD9 and BCL10 were successfully purified and characterized, and their biochemical properties were investigated. In addition, CARD9-BCL10 complexes were reconstituted in vitro under low salt and $\mathrm{pH}$ conditions. Furthermore, based on structural modeling data, a scheme was proposed to describe the interactions between CARD9 and BCL10.

\section{Materials and methods}

Materials. DNA restriction enzymes (NdeI and XhoI), T4 DNA ligase and Taq DNA polymerase were obtained from New England BioLabs, Inc. (Ipswich, MA, USA). Isopropyl $\beta$-D-1-thiogalactopyranoside (IPTG), urea, imidazole, and dithiothreitol were purchased from Sigma-Aldrich; Merck KGaA (Darmstadt, Germany). E. coli BL21 (DE3) cells and plasmid pET expression vectors were obtained from Novagen; Merck KGaA. A Superdex 200 gel filtration column was purchased from GE Healthcare Life Sciences (Chalfont, UK). A protease inhibitor cocktail was acquired from Roche Diagnostics (Indianapolis, IN, USA). Concentrators were obtained from Merck KGaA.

Expression of CARD9 and BCL1O CARDs in E. coli cells. cDNAs of full length mouse CARD9 (amino acids, 1-536) and BCL10 (amino acids, 1-233), obtained from Harvard University (Cambridge, MA, USA) were used as templates for polymerase chain reaction (PCR), while plasmid expression vectors pET28a and pET26b were used to add a hexahistidine tag to the $\mathrm{N}$ - and C-termini of CARD9 and the C-terminus of BCL10 for affinity purification. The CARD of human CARD9, corresponding to amino acids 1-100, was cloned into pET28a, resulting in pET (6His-CARD9 CARD-6His). The CARD of BCL10, corresponding to amino acids 1-93 was cloned into pET26b, resulting in pET (BCL10 CARD-6His).

Each clone was transformed into $E$. coli via heat shock at $42^{\circ} \mathrm{C}$ and was streaked onto Luria Bertani medium (LB; Sigma-Aldrich; KGaA Millipore) Agar plates containing the appropriate antibiotics $(100 \mu \mathrm{l} / \mathrm{ml}$ ampicillin for the $\mathrm{pET} 26 \mathrm{~b}$ clone and $50 \mu \mathrm{l} / \mathrm{ml}$ kanamycin for the pET28a clone), and plates were incubated at $37^{\circ} \mathrm{C}$ for $18 \mathrm{~h}$. A single colony was used to inoculate $5 \mathrm{ml}$ of LB medium (Sigma-Aldrich; KGaA Millipore), which was subsequently incubated at $37^{\circ} \mathrm{C}$ under agitation overnight. A total of $5 \mathrm{ml}$ of the small scale culture was used to inoculate $1,000 \mathrm{ml}$ of LB medium in a 2,000 ml culture flask, and the culture was incubated at $37^{\circ} \mathrm{C}$ under agitation for $\sim 4 \mathrm{~h}$. When the optical density at $600 \mathrm{~nm}$ was between 0.6 and 0.7 , expression was induced via overnight incubation at $20^{\circ} \mathrm{C}$ with $0.5 \mathrm{mM}$ IPTG.
Purification of target proteins. E. coli cells expressing the proteins were centrifuged at $3,000 \times \mathrm{g}$ for $20 \mathrm{~min}$ at $4{ }^{\circ} \mathrm{C}$, resuspended and lysed by sonication in $50 \mathrm{ml}$ of lysis buffer, containing $20 \mathrm{mM}$ sodium citrate (pH 5.0), $500 \mathrm{mM} \mathrm{NaCl}$ and $5 \mathrm{mM}$ imidazole, supplemented with protease inhibitors (Sigma-Aldrich; KGaA Millipore). Lysates were centrifuged at $11,000 \mathrm{x} \mathrm{g}$ for $1 \mathrm{~h}$ at $4^{\circ} \mathrm{C}$ and supernatants were isolated and applied to a gravity column (Bio-Rad Laboratories, Inc., Hercules, CA, USA) packed with Ni-NTA affinity resin (Qiagen China Co., Ltd., Shanghai, China). Unbound bacterial proteins were removed using $100 \mathrm{ml}$ of washing buffer, containing $20 \mathrm{mM}$ Tris buffer $(\mathrm{pH} 8.0), 500 \mathrm{mM} \mathrm{NaCl}$ and $60 \mathrm{mM}$ imidazole. Target proteins were eluted from the column using elution buffer, containing $20 \mathrm{mM}$ sodium citrate ( $\mathrm{pH}$ 5.0), $500 \mathrm{mM} \mathrm{NaCl}$ and $250 \mathrm{mM}$ imidazole, with $1 \mathrm{ml}$ elution fractions being collected over a total of $10 \mathrm{ml}$. Fractions containing $>90 \%$ homogeneous target protein, following separation by $15 \%$ SDS-PAGE were selected and combined. SDS-PAGE was visualized by coomassie staining (Bio-Rad Laboratories, Inc.). For final purification, the sample was loaded onto a Superdex 200 gel filtration column and equilibrated with the aforementioned buffers. Fractions containing the target proteins were pooled and maintained at $4^{\circ} \mathrm{C}$. Protein homogeneity was assessed by $15 \%$ SDS-PAGE, followed by visualization via coomassie staining (Bio-Rad Laboratories, Inc.).

Circular dichroism spectroscopy. Protein secondary structures were investigated using circular dichroism (CD) spectroscopy with a Jasco J-715 spectropolarimeter (JASCO International Co. Ltd., Tokyo, Japan). Spectra were obtained at wavelengths between $200-250 \mathrm{~nm}$ at $25^{\circ} \mathrm{C}$ in a $0.1 \mathrm{~cm}$ path-length quartz cuvette, using a bandwidth of $1.0 \mathrm{~nm}$, a speed of $50 \mathrm{~mm} / \mathrm{min}$, and a response time of $5 \mathrm{sec}$. Protein samples were maintained in buffer containing $20 \mathrm{mM}$ Tris- $\mathrm{HCl}(\mathrm{pH} 8.0)$ and $150 \mathrm{mM}$ $\mathrm{NaCl}$, and were diluted to $0.1 \mathrm{mg} / \mathrm{ml}$ prior to analysis. Four scans were conducted and averaged, and the $\alpha$-helical content was calculated using the molar ellipticity at $222 \mathrm{~nm}$ (27). Percentage of helical content was estimated using the Advanced Protein Secondary Structure Prediction Server for protein secondary structure prediction (www.imtech.res. in/raghava/apssp/).

Gel filtration chromatography. Purified CARDs from CARD9 and BCL10 were incubated together for $1 \mathrm{~h}$ at room temperature. Following incubation, the mixture was concentrated to $10-15 \mathrm{mg} / \mathrm{ml}$ using a concentrator. The concentrated protein mixture was applied to a Superdex 200 10/300 gel filtration column, that had been pre-equilibrated under specific buffer conditions. ACTA_FPLC (GE Healthcare Life Sciences), was used for chromatography. Complex assembly was evaluated based on the positions of the eluted peaks, monitored at a wavelength of $280 \mathrm{~nm}$ and followed by $15 \%$ SDS-PAGE, visualized by coomassie staining (Bio-Rad Laboratories, Inc.).

Native PAGE. Formation of the complex between CARD9 and BCL10 CARDs was assessed using native (non-denaturing) PAGE on an Amersham ${ }^{\mathrm{TM}}$ PhastSystem (GE Healthcare Life Sciences) with pre-made $8-25 \%$ acrylamide gradient gels (GE Healthcare Life Sciences). Coomassie Brilliant Blue dye was used for the detection of protein band patterns. Separately 
purified CARD9 and BCL10 CARDs were pre-incubated together for $1 \mathrm{~h}$ at room temperature and the mixture was applied to the gel. Complex assembly was evaluated based on the appearance of newly formed bands and disappearance of existing bands.

Homology modeling. Homology models of CARD9 and BCL10 CARDs were constructed using the SWISS-MODEL homology modeling server (http://swissmodel.expasy .org) (28). The caspase-9 CARD structure (Protein Data Bank ID, 3YGS) and the receptor interacting protein-associated ICH-1 homologous protein with a death domain (RAIDD) CARD structure (Protein Data Bank ID, 3CRD) were used as the modeling templates for CARD9 and BCL10 CARDs, respectively. The stereochemical quality of the constructed models was validated using a Ramachandran plot generated by PROCHECK (http://www.ebi.ac.uk/thornton-srv/software/PROCHECK/) (29). Ribbon diagrams were generated using PyMOL software (Schrödinger, LLC, Tokyo, Japan) (30).

\section{Results and Discussion}

Expression, purification and characterization of CARD 9 CARD. The CARD9 signalosome, which is composed of CARD9, BCL10 and MALT1, serves a critical role in innate immunity through the regulation of immune cell-mediated NF- $\kappa$ B activation. CARD9, BCL10 and MALT1 contain structures belonging to the death fold class of protein motifs at their N-terminus, which enable homotypic protein-protein interactions; CARD9 and BCL10 contain CARD, whereas MALT1 contains a DD (Fig. 1A).

In order to elucidate the molecular basis of CARD9 signalosome assembly, CARD9 and BCL10 CARDs were purified. Soluble BCL10 CARD was purified using two step affinity chromatography, followed by gel filtration chromatography (Fig. 1B). DD-containing proteins are difficult to purify in their functional form, due to their insolubility (24-26); notably, in the present study, following IPTG induction, $E$. coli cells (BL21-DE3 strain) transfected with CARD9 CARD (amino acids, 1-100) using a pET28a expression vector, were demonstrated to express the recombinant protein, which was detected in the soluble fraction. Various buffer conditions were used during purification to identify the optimal conditions for CARD9 CARD stabilization. Gel filtration chromatography profiles revealed that CARD9 CARD was stable at $\mathrm{pH} 8.0$ and salt concentration of $150 \mathrm{mM}$ following an elution of $16 \mathrm{ml}$ from the gel filtration column (Fig. 1C and D). At pH 5.0 in salt-free solution, CARD9 CARD was eluted with an unusual pattern. Overall, the effects of $\mathrm{pH}$ and salt concentration on CARD9 CARD solubility were in accordance with previous studies, and indicated that the solubility of DD-containing proteins is sensitive to $\mathrm{pH}$ and salt concentration $(11,12,31,32)$.

Structures of purified CARDs are typical $\alpha$-helical bundle folds. To confirm that purified CARD9 and BCL10 CARDs retained their physiological secondary structure, far-UV CD spectra were evaluated. CARD9 and BCL10 CARDs exhibited CD spectral patterns typical of $\alpha$-helical proteins, characterized by two pronounced minimums at 208 and $222 \mathrm{~nm}$ and a maximum at $195 \mathrm{~nm}$ (Fig. 2), similar to molecular structures of other DD-containing proteins (31). In addition, the measured percentage of helical content agreed with the predicted values (BCL10 CARD, $56 \%$ predicted vs. 58\% measured; CARD9 CARD, $52 \%$ predicted vs. $56 \%$ measured; Fig. 2).

Purified CARD9 and BCL1O CARDs interact in vitro under low salt and $\mathrm{pH}$ conditions. During CARD9 signalosome assembly, CARD9 and BCL10 CARDs have been reported to interact directly $(33,34)$. To determine whether purified CARD9 and BCL10 CARDs maintained their ability to interact in vitro, separately purified CARD9 and BCL10 CARDs were incubated together and subjected to gel filtration chromatography. Complex assembly was evaluated based on the eluted peaks monitored at $280 \mathrm{~nm}$, followed by SDS-PAGE. Due to the sensitivity of DD-containing protein solubility to salt concentration and $\mathrm{pH}$, analysis was performed under the following salt and $\mathrm{pH}$ conditions: In $\mathrm{pH} 8.0$ without salt (Fig. 3A); in pH 5.0 without salt (Fig. 3B); in pH 8.0 with $150 \mathrm{mM} \mathrm{NaCl}$ (Fig. 3C); and in pH 8.0 with $1 \mathrm{M} \mathrm{NaCl}$ (Fig. 3D). New distinct peaks were not generated under any of the aforementioned conditions; however, co-migration of CARD9 and BCL10 CARDs on SDS-PAGE was detected at pH 5.0 without salt (Fig. 3B). A 1:1 pattern of co-migration on SDS-PAGE may indicate that CARD9 and BCL10 CARDs can interact in vitro. Native PAGE was used to confirm the in vitro interaction between CARD9 BCL10 CARDs (Fig. 4A). The CARD9 and BCL10 CARD mixture produced smeared complex bands, indicating that the CARDs may interact in vitro (Fig. 4A). Separately purified CARD9 and BCL10 CARDs produced single bands following SDS-PAGE (Fig. 4B).

A model of the interaction between CARD9 and BCL1O $C A R D s$. DD-containing proteins have been reported to form two classes of complexes, dimeric and helical oligomeric complexes (12). Dimeric complexes have been reported to be formed through the interaction of DDs of the adaptor protein Tube and the kinase Pelle, as well as the interaction of CARDs of apoptotic protease activating factor 1 (Apaf-1) and caspase-9 (14). Helical oligomeric complexes have been reported to be formed through the interaction of DDs of RAIDD and p53-induced death domain protein 1, of Fas and Fas-associated protein with death domain, and of IL-1 receptor-associated kinase 2 (IRAK2), IRAK4 and myeloid differentiation primary response gene $88(14,26)$. Since CARD-CARD interactions have been demonstrated to mediate the formation of various signaling complexes, including the CARD signalosome, it is of great interest to elucidate the structural aspects of CARD-CARD interactions; however, only the crystal structure of the Apaf-1 CARD-caspase-9 CARD complex has been solved to date (35). Due to the lack of structural information regarding CARD complexes, the ability of CARDs to form helical assemblies, as well as their stoichiometry during CARD9 signalosome assembly, has yet to be elucidated. Further biochemical and structural studies are required to address these questions and understand the molecular basis of CARD-mediated interactions during CARD9 signalosome assembly.

In the present study, due to the lack of structural information regarding the CARD9 CARD-BCL10 CARD complex, 
A CARD9

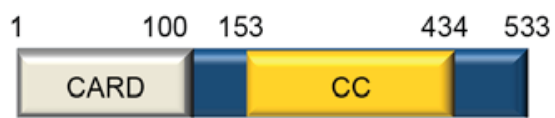

BCL10

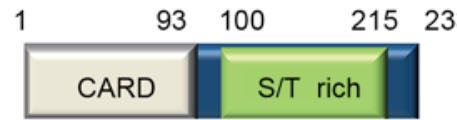

MALT1

\begin{tabular}{|l|l|l|l|l|l|}
132 & 139 & 203 & 306 & 356 & 570 \\
\hline DD & IG & IG & & Caspase & \\
\hline
\end{tabular}

C

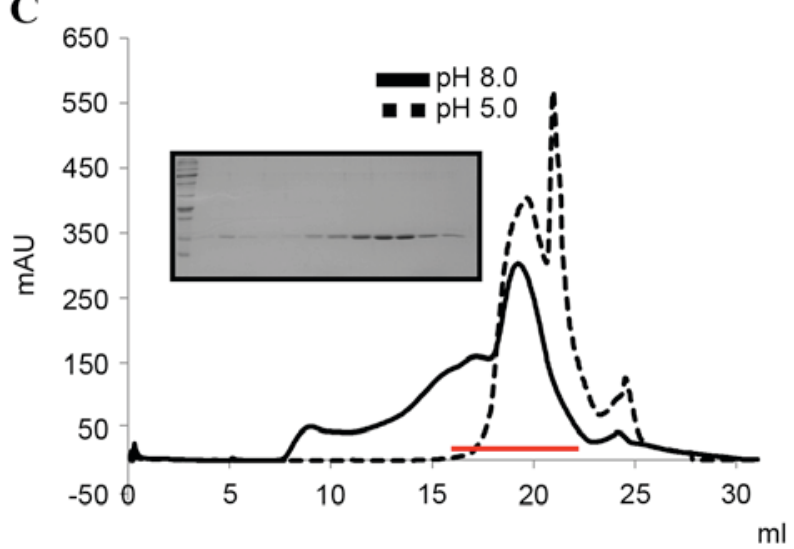

[CARD9 CARD]

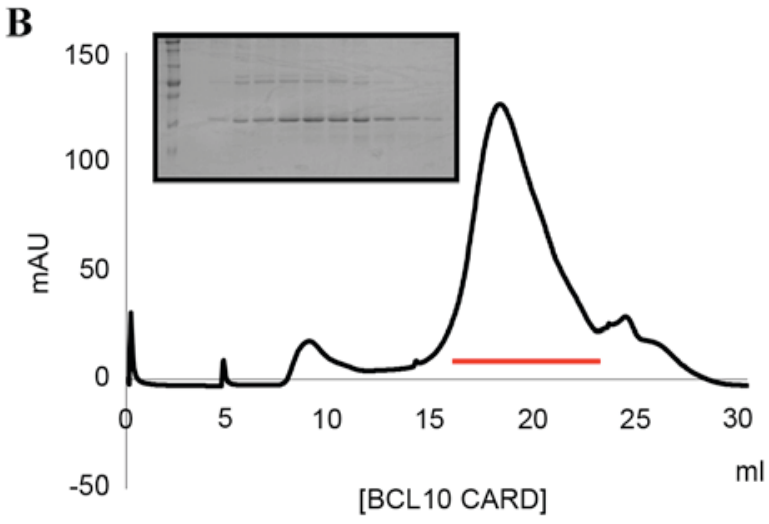

D

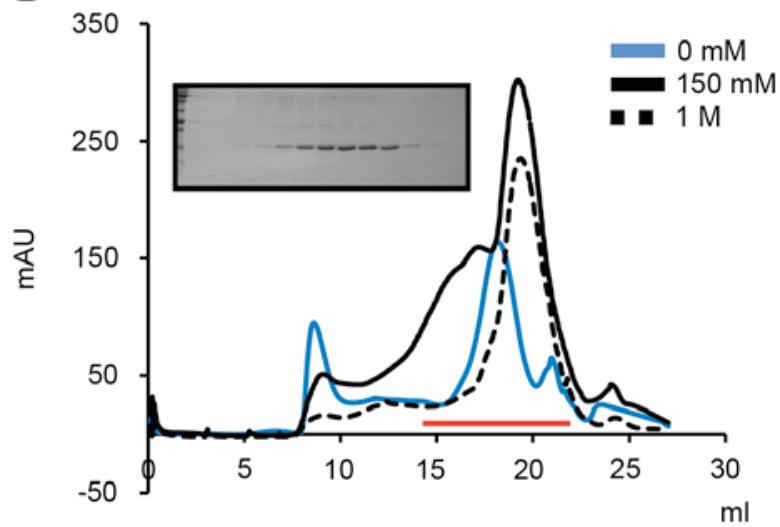

[CARD9 CARD]

Figure 1. Purification of CARD9 and BCL10 CARDs. (A) Domains identified in the CARD9, BCL10 and MALT1 proteins of the CARD9 signalosome. The death fold motifs CARD and DD are presented in gray. Amino acid residues corresponding to the various domains are indicated. (B) Purification of BCL10 CARD was performed using gel filtration chromatography. Gel filtration chromatography profiles were obtained for the purpose of biochemical characterization of CARD9 CARD in vitro under different (C) pH and (D) salt conditions. Eluted fractions were separated by SDS-PAGE and stained using Coomassie Blue at (C) pH 8.0 and (D) $150 \mathrm{mM} \mathrm{NaCl}$. Red bars indicate the fractions subjected to SDS-PAGE. CARD9, CARD-containing protein 9; BCL10, B-cell CLL/lymphoma 10; CARD, caspase-associated recruitment domain; MALT, mucosa-associated lymphoid tissue lymphoma translocation protein; DD, death domain; CC, coiled-coil; IG, immunoglobulin; S/T, Ser/Thr.

A

[BCL10 CARD]

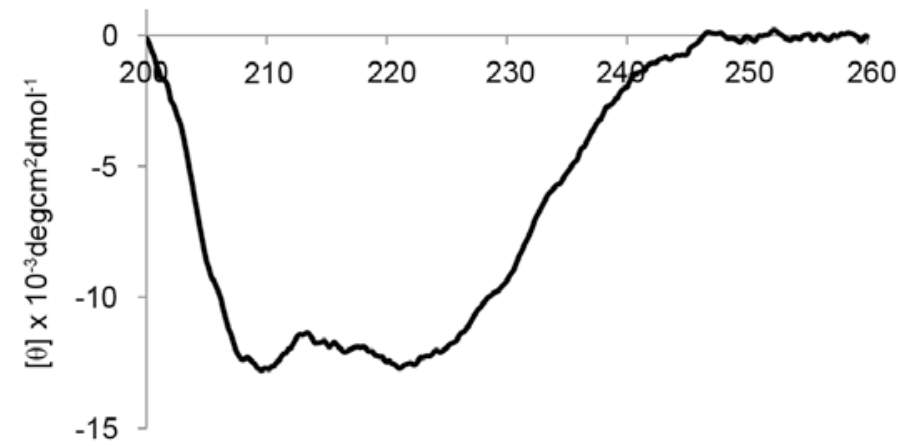

Wavelength, $\mathrm{nm}$

$\%$ of helix contents predicted: 56

$\%$ of helix contents measured: 58
B

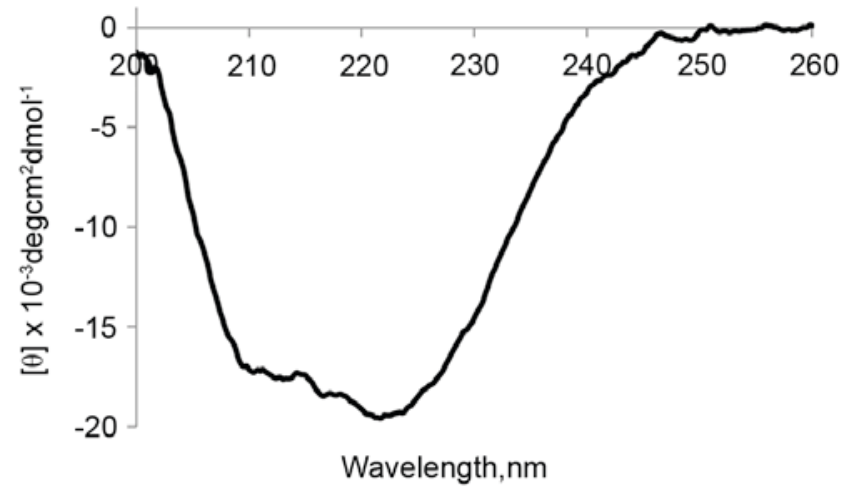

$\%$ of helix contents predicted: 52

$\%$ of helix contents measured: 56

Figure 2. Circular dichroic spectra of purified (A) BCL10 CARD and (B) CARD9 CARD. Spectra were recorded at $25^{\circ} \mathrm{C}$. Four independent scans were conducted using a Jasco J-715 spectropolarimeter and averaged. BCL10, B-cell CLL/lymphoma 10; CARD, caspase-associated recruitment domain; CARD9, CARD-containing protein 9.

estimates of the interaction mode were based on sequence analysis and structural modeling data. Homology modeling of the CARD9 CARD was performed using the caspase-9 CARD structure (Protein Data Bank ID, 3YGS) in order to 

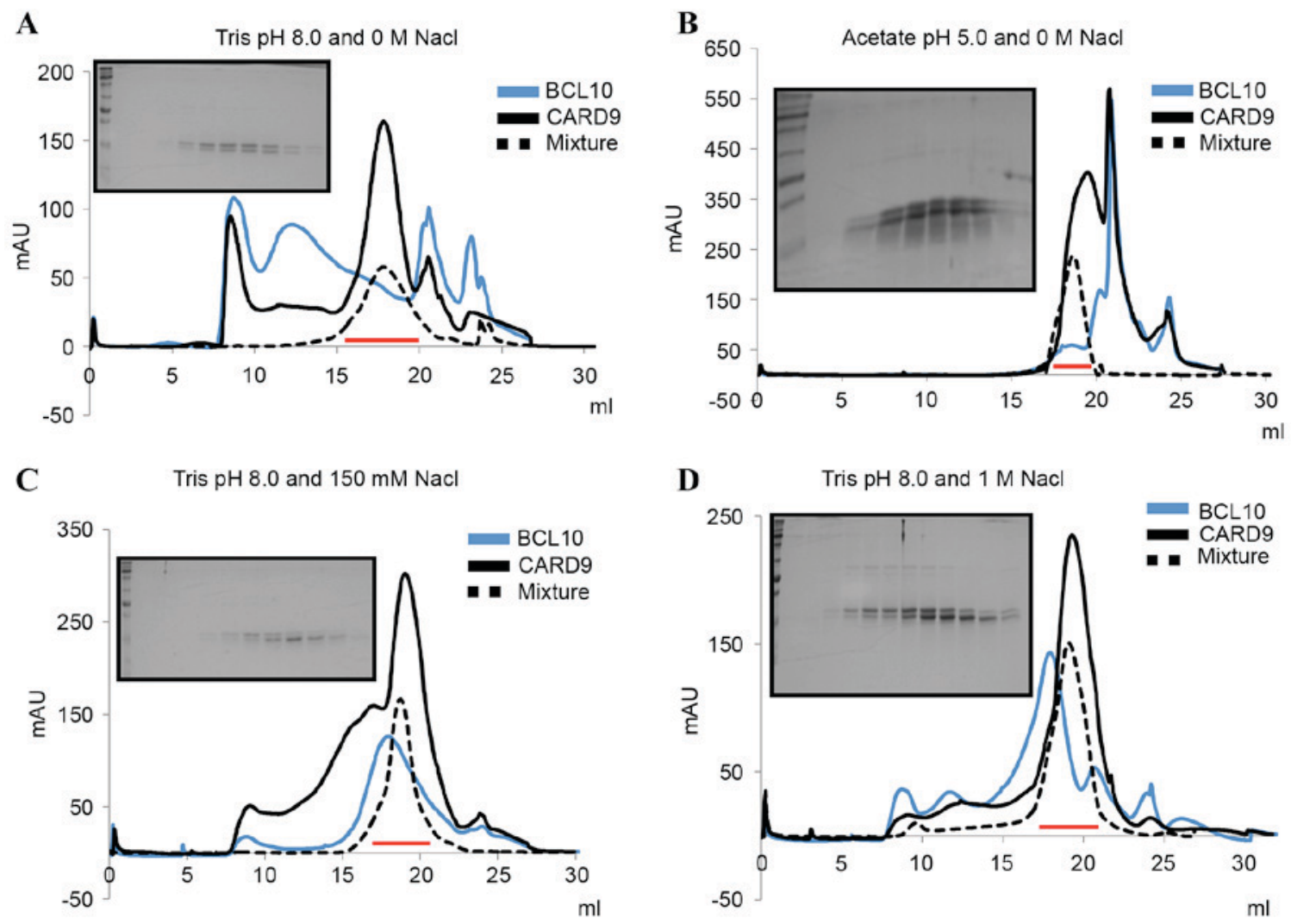

Figure 3. Complex formation was investigated using gel filtration chromatography. Separately purified CARD9 and BCL10 CARDs were incubated together at room temperature for $1 \mathrm{~h}$ and loaded onto gel filtration columns. Columns were pre-equilibrated in buffer containing (A) $20 \mathrm{mM} \mathrm{Tris-HCl}(\mathrm{pH} 8.0)$ and $0 \mathrm{M}$ $\mathrm{NaCl}$, (B) $20 \mathrm{mM}$ Acetate (pH 5.0) and $0 \mathrm{M} \mathrm{NaCl}$, (C) $20 \mathrm{mM}$ Tris- $\mathrm{HCl}$ (pH 8.0) and $150 \mathrm{mM} \mathrm{NaCl}$ and (D) $20 \mathrm{mM}$ Tris-HCl pH 8.0 and $1 \mathrm{M} \mathrm{NaCl}$. Eluted fractions were separated by SDS-PAGE and representative gels are included. Red bars indicate the fractions subjected to SDS-PAGE. CARD9, CARD-containing protein 9; BCL10, B-cell CLL/lymphoma 10; CARD, caspase-associated recruitment domain.

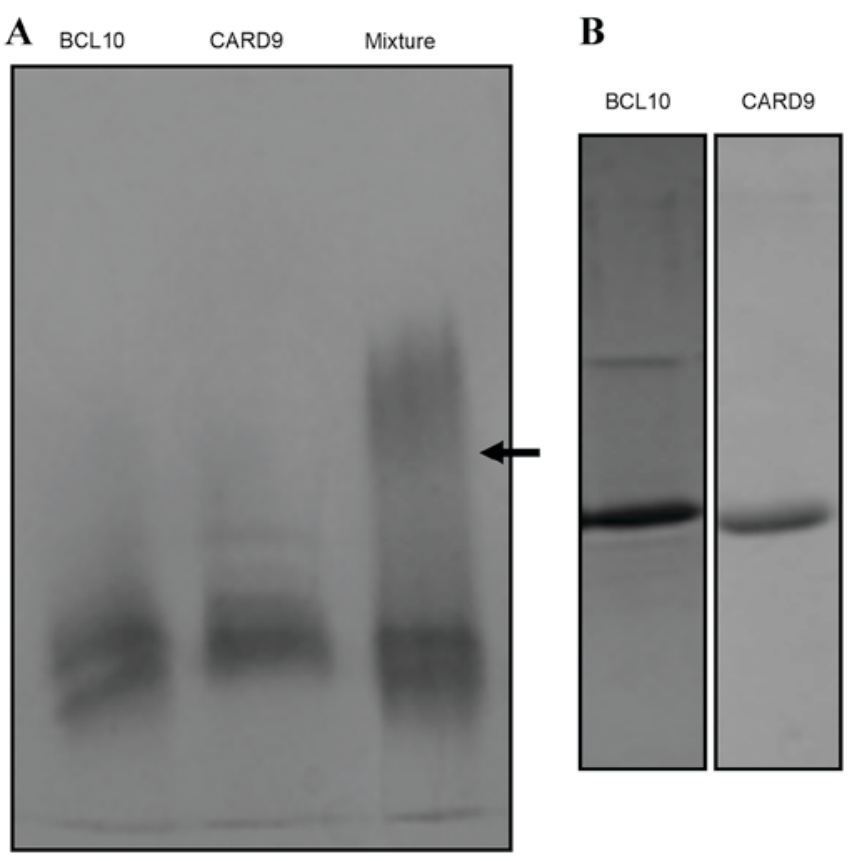

Figure 4. Complex formation was investigated using native PAGE. (A) Native PAGE of the specific interactions between BCL10 and CARD9 CARDs. Black arrows indicate newly formed complex bands. (B) SDS-PAGE of purified BCL10 and CARD9 CARDs. BCL10, B-cell CLL/lymphoma 10; CARD9, CARD-containing protein 9; CARD, caspase-associated recruitment domain. investigate whether the interaction between CARD9 and BCL10 CARDs during CARD9 signalosome assembly resembled the interaction between Apaf-1 and caspase-9 CARDs during apoptosome assembly (35). The CARD9 CARD exhibited $16 \%$ sequence homology with the caspase-9 CARD (Fig. 5A). It has previously been reported that the Apaf-1 CARD-caspase-9 CARD complex involved mainly hydrogen bonds and van der Waals interactions (35). Three positively charged residues in caspase-9 CARD, i.e., R13, R52 and R56, and two negatively charged residues in Apaf-1 CARD, i.e., D27 and E40, were reported to be crucial for interaction (35). Based on sequence alignment, two of the positively charged residues of the caspase-9 CARD appeared to be conserved in CARD9 and BCL10 CARD sequences (blue residues; Fig. 5B). The negatively charged residues D27 and E40, were conserved in the BCL10 CARD, whereas only E40 was conserved in the CARD9 CARD (red residues; Fig. 5B). In the present study, structural models of the CARD9 CARD were well constructed and contained six $\alpha$-helical bundles, which have been identified as the typical structural composition of DD-containing proteins (Fig. 5C) (35). A previously generated model of the BCL10 CARD was used in the present study (36). To investigate the putative interaction interface, model structures of CARD9 and BCL10 CARDs were superimposed with the previously solved Apaf-1 CARD-caspase-9 CARD complex structure (PDBid: 3YGS; Fig. 5D and E) (35). Two 
A
CARD 9
Casp-9
CARD9
Casp-9

$\begin{array}{ll}5 & \text { ENDDECWSTLESFRVKLISVIDPSRITPYLRQCKVLNPDDEEQVLSDPNLVIRKRKVGVL } 64 \\ 1 \text { SMDEADRRLLRRCRLRLVEELQVDQLWDVLLSRELFRPHMIEDIQRAG-SGSRRDQARQL } 59\end{array}$

B

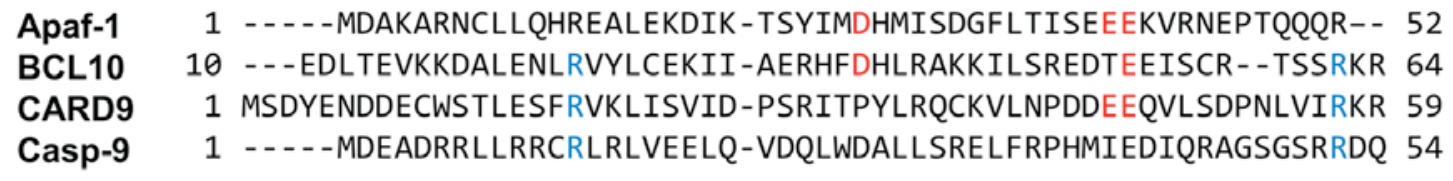

Apaf-1 53 -AAMLIKMILKKDNDSYVSFYNALLHEGYKDLAALLHDG - . - . - 90

BCL10 65 AGK-LLDYLQEN-PRGLDTLVESIRREKTQSFLIQKITDEVLKLRNIK 110

CARD9 60 KVGVLLDILQRTGHKGYVAFLESLELYYPQ--LYRKVTGKEPA--.-- 100

Casp-9 55 ARQLIIDLETRG-SQALPLFISCLEDTGQDMLASFLRTN-.....-. 92

C

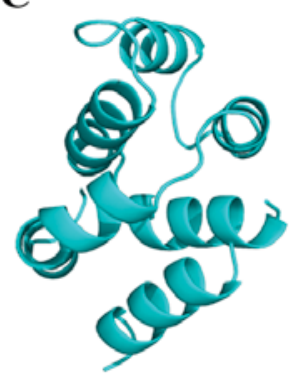

CARD9

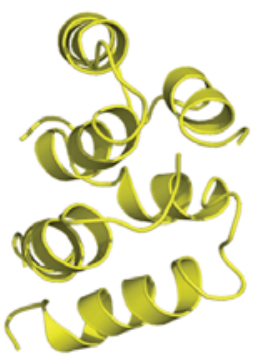

BCL10
D

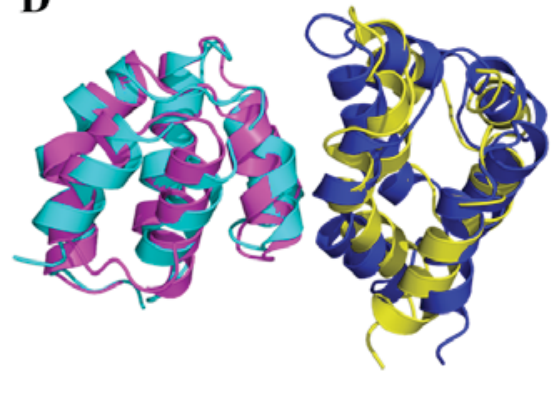

CARD9/Apaf-1
$\mathbf{E}$

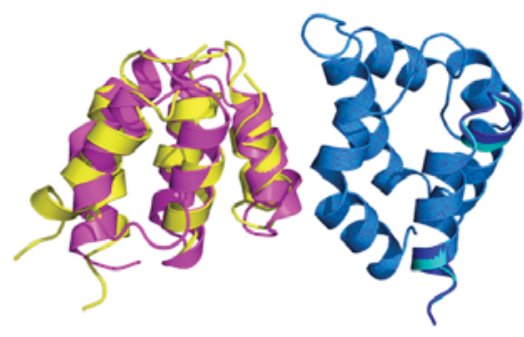

BCL10/Apaf-1

CARD9/Casp9

Figure 5. Homology modeling of the CARD9 CARD structure and proposed mode of interaction between BCL10 and CARD9 CARDs. (A) Sequence alignment between CARD9 and caspase-9 CARDs, with identical bases shown in bold font. A total of $16 \%$ sequence identity was revealed and the caspase-9 CARD was used as a template for homology modeling of the CARD9 CARD structure. (B) Sequence alignment between Apaf-1, BCL10 and CARD9 CARDs, and caspase-9. Residues critical for the Apaf-1 CARD-caspase-9 CARD interaction and conserved residues on BCL10 and CARD9 CARDs are presented in red or blue. Red and blue indicate acidic and basic residues, respectively. (C) Structural model of the CARD9 CARD predicted using SWISS-MODEL, based on the template structure of the caspase-9 CARD (Protein Data Bank ID, 3YGS). The structural model of the BCL10 CARD, as previously predicted using SWISS-MODEL (36), based on the template structure of the RAIDD CARD (Protein Data Bank ID, 3CRD) is also presented. (D and E) Superimposition of BCL10 and CARD9 CARDs with the Apaf-1 CARD-caspase-9 CARD complex. CARD9 CARD (cyan) was superimposed with (D) Apaf-1 CARD (magenta) or (E) caspase-9 CARD (blue). BCL10 CARD (yellow) was superimposed with (D) caspase-9 CARD (blue) or (E) Apaf-1 CARD (magenta). CARD9, CARD-containing protein 9; CARD, caspase-associated recruitment domain; BCL10, B-cell CLL/lymphoma 10; Casp9, caspase-9; Apaf-1, apoptotic protease activating factor 1; RAIDD, receptor interacting protein-associated ICH-1 homologous protein with a death domain.

possibilities, i.e., CARD9/Apaf-1-BCL10/caspase-9 (Fig. 5D) or BCL10/Apaf-1-CARD9/caspase-9 (Fig. 5E), were considered. Based on sequence alignment data and the structure of the Apaf-1 CARD-caspase-9 CARD complex, the present results suggested that three positively charged residues of the BCL10 CARD, i.e., R25, R62 and K67, which were conserved in the caspase-9 CARD (R13, R52 and R56), as well as one negatively charged residue of the CARD9 CARD (E46), which was conserved in the Apaf-1 CARD (E40), may be crucial for the CARD9 CARD-BCL10 CARD interaction, provided that the complex is formed as: CARD9/Apaf-1-BCL10/caspase-9 (Fig. 5D). Conversely, if the complex is formed as: BCL10/Apaf-1-CARD9/caspase-9 (Fig. 5E), two conserved residues on the BCL10 CARD, i.e., D38 and E52, and two conserved residues on the CARD9 CARD, i.e., R18 and R57, may be critical for complex formation. The results of the present study, suggesting the formation of a hetero-dimeric CARD9 CARD-BCL10 CARD complex, are in accordance with previous studies demonstrating the formation of dimeric CARD complexes. However, other proteins containing structures belonging to the death fold class of motifs, such as DED and PYD, may form more complicated oligomeric complexes (17,37-39).

In conclusion, a hetero-dimeric CARD complex between CARD9 CARD and BCL10 CARD was successfully reconstituted in vitro and the complex was modelled based on the previously available CARD structure. The present study demonstrated that the CARD9-BCL10 complex may use a typical CARD-CARD interaction strategy for assembly. This provides a further understanding of the mechanism of how the CARD9 signalosome may be assembled via a CARD-CARD interaction. 


\section{Acknowledgements}

The present study was supported by Yeungnam University in 2014.

\section{References}

1. Malinin NL, Boldin MP, Kovalenko AV and Wallach D: MAP3K-related kinase involved in NF-kappaB induction by TNF, CD95 and IL-1. Nature 385: 540-544, 1997.

2. Alexopoulou L, Holt AC, Medzhitov R and Flavell RA: Recognition of double-stranded RNA and activation of NF-kappaB by Toll-like receptor 3. Nature 413: 732-738, 2001.

3. Baeuerle PA and Baltimore D: NF-kappa B: Ten years after. Cell 87: 13-20, 1996.

4. Baeuerle PA and Henkel T: Function and activation of NF-kappa B in the immune system. Annu Rev Immunol 12: 141-179, 1994.

5. Li Q and Verma IM: NF-kappaB regulation in the immune system. Nat Rev Immunol 2: 725-734, 2002.

6. Thome M: CARMA1, BCL-10 and MALT1 in lymphocyte development and activation. Nat Rev Immunol 4: 348-359, 2004.

7. Hara H, Ishihara C, Takeuchi A, Imanishi T, Xue L, Morris SW, Inui M, Takai T, Shibuya A, Saijo S, et al: The adaptor protein CARD9 is essential for the activation of myeloid cells through ITAM-associated and Toll-like receptors. Nat Immunol 8: 619-629, 2007

8. Hsu YM, Zhang Y, You Y, Wang D, Li H, Duramad O, Qin XF, Dong $\mathrm{C}$ and $\mathrm{Lin} \mathrm{X}$ : The adaptor protein CARD9 is required for innate immune responses to intracellular pathogens. Nat Immunol 8: 198-205, 2007.

9. Hara $\mathrm{H}$ and Saito T: CARD9 versus CARMA1 in innate and adaptive immunity. Trends Immunol 30: 234-242, 2009.

10. Hara H, Wada T, Bakal C, Kozieradzki I, Suzuki S, Suzuki N, Nghiem M, Griffiths EK, Krawczyk C, Bauer B, et al: The MAGUK family protein CARD11 is essential for lymphocyte activation. Immunity 18: 763-775, 2003.

11. Bae JY and Park HH: Crystal structure of NALP3 protein pyrin domain (PYD) and its implications in inflammasome assembly. J Biol Chem 286: 39528-39536, 2011.

12. Park HH: Structural analyses of death domains and their interactions. Apoptosis 16: 209-220, 2011.

13. Park HH: Structural features of caspase-activating complexes. Int J Mol Sci 13: 4807-4818, 2012.

14. Park HH, Lo YC, Lin SC, Wang L, Yang JK and Wu H: The death domain superfamily in intracellular signaling of apoptosis and inflammation. Ann Rev Immunol 25: 561-586, 2007.

15. Weber $\mathrm{CH}$ and Vincenz C: The death domain superfamily: A tale of two interfaces? Trends Biochem Sci 26: 475-481, 2001.

16. Reed JC, Doctor KS and Godzik A: The domains of apoptosis: A genomics perspective. Sci STKE 2004: re9, 2004

17. Park HH, Logette E, Rauser S, Cuenin S, Walz T, Tschopp J and $\mathrm{Wu} \mathrm{H}$ : Death domain assembly mechanism revealed by crystal structure of the oligomeric PIDDosome core complex. Cell 128 : 533-546, 2007.

18. Zhou H, Wertz I, O'Rourke K, Ultsch M, Seshagiri S, Eby M, Xiao W and Dixit VM: Bcl10 activates the NF-kappaB pathway through ubiquitination of NEMO. Nature 427: 167-171, 2004.

19. Oeckinghaus A, Wegener E, Welteke V, Ferch U, Arslan SC, Ruland J, Scheidereit C and Krappmann D: Malt1 ubiquitination triggers NF-kappaB signaling upon T-cell activation. EMBO J 26: 4634-4645, 2007.

20. Rebeaud F, Hailfinger S, Posevitz-Fejfar A, Tapernoux M, Moser R, Rueda D, Gaide O, Guzzardi M, Iancu EM, Rufer N, et al: The proteolytic activity of the paracaspase MALT1 is key in T cell activation. Nat Immunol 9: 272-281, 2008.
21. Coornaert B, Baens M, Heyninck K, Bekaert T, Haegman M, Staal J, Sun L, Chen ZJ, Marynen P and Beyaert R: T cell antigen receptor stimulation induces MALT1 paracaspase-mediated cleavage of the NF-kappaB inhibitor A20. Nat Immunol 9: 263-271, 2008.

22. Uren AG, O'Rourke K, Aravind LA, Pisabarro MT, Seshagiri S, Koonin EV and Dixit VM: Identification of paracaspases and metacaspases: Two ancient families of caspase-like proteins, one of which plays a key role in MALT lymphoma. Mol Cell 6: 961-967, 2000.

23. Lucas PC, Yonezumi M, Inohara N, McAllister-Lucas LM, Abazeed ME, Chen FF, Yamaoka S, Seto M and Nunez G: Bcl10 and MALT1, independent targets of chromosomal translocation in malt lymphoma, cooperate in a novel NF-kappa B signaling pathway. J Biol Chem 276: 19012-19019, 2001.

24. Huang B, Eberstadt M, Olejniczak ET, Meadows RP and Fesik SW: NMR structure and mutagenesis of the Fas (APO-1/CD95) death domain. Nature 384: 638-641, 1996.

25. Jang TH and Park HH: Purification, crystallization and preliminary x-ray crystallographic studies of RAIDD Death-Domain (DD). Int J Mol Sci 10: 2501-2509, 2009.

26. Jeong EJ, Bang S, Lee TH, Park YI, Sim WS and Kim KS: The solution structure of FADD death domain. Structural basis of death domain interactions of Fas and FADD. J Biol Chem 274: 16337-16342, 1999.

27. Chen YH, Yang JT and Martinez HM: Determination of the secondary structures of proteins by circular dichroism and optical rotatory dispersion. Biochemistry 11: 4120-4131, 1972.

28. Schwede T, Kopp J, Guex N and Peitsch MC: SWISS-MODEL: An automated protein homology-modeling server. Nucleic Acids Res 31: 3381-3385, 2003.

29. Laskowski RA, Rullmannn JA, MacArthur MW, Kaptein R and Thornton JM: AQUA and PROCHECK-NMR: Programs for checking the quality of protein structures solved by NMR J Biomol NMR 8: 477-486, 1996.

30. DeLano WL: The PyMOL Molecular Graphics System. DeLano Scientific, San Carlos, CA, 2002.

31. Bae JY and Park HH: Crystallization and preliminary X-ray crystallographic studies of the PYD domain of human NALP3. Acta Crystallogr Sect F Struct Biol Cryst Commun 67: 1421-1424, 2011.

32. Jang TH and Park HH: Generalized semi-refolding methods for purification of the functional death domain superfamily. J Biotechnol 151: 335-342, 2011.

33. Wegener E and Krappmann D: CARD-Bcl10-Malt1 signalosomes: Missing link to NF-kappaB. Sci STKE 2007: pe21, 2007.

34. Liu Y, Song R, Gao Y, Li Y, Wang S, Liu HY, Wang Y, Hu YH and Shu HB: Protein kinase $\mathrm{C}-\delta$ negatively regulates $\mathrm{T}$ cell receptor-induced NF- $\kappa \mathrm{B}$ activation by inhibiting the assembly of CARMA1 signalosome. J Biol Chem 287: 20081-20087, 2012.

35. Qin H, Srinivasula SM, Wu G, Fernandes-Alnemri T, Alnemri ES and Shi Y: Structural basis of procaspase-9 recruitment by the apoptotic protease-activating factor 1. Nature 399: 549-557, 1999.

36. Park JH, Bae JY and Park HH: Self-oligomerization of the CARD domain prevents complex formation in the CARMA1 signalosome. Int J Mol Med 31: 1280-1287, 2013.

37. Park HH: PYRIN domains and their interactions in the apoptosis and inflammation signaling pathway. Apoptosis 17: 1247-1257, 2012.

38. Wang L, Yang JK, Kabaleeswaran V, Rice AJ, Cruz AC, Park AY, Yin Q, Damko E, Jang SB, Raunser S, et al: The Fas-FADD death domain complex structure reveals the basis of DISC assembly and disease mutations. Nat Struct Mol Biol 17: 1324-1329, 2010.

39. Scott FL, Stec B, Pop C, Dobaczewska MK, Lee JJ, Monosov E, Robinson H, Salvesen GS, Schwarzenbacher R and Riedl SJ: The Fas-FADD death domain complex structure unravels signalling by receptor clustering. Nature 457: 1019-1022, 2009. 